Article

\title{
Lactobacillus Acidophilus/Bifidobacterium Infantis Probiotics Are Beneficial to Extremely Low Gestational Age Infants Fed Human Milk
}

\author{
Ingmar Fortmann ${ }^{1, *(\mathbb{D})}$, Janina Marißen ${ }^{1}$, Bastian Siller ${ }^{1}$, Juliane Spiegler ${ }^{1} \mathbb{D}$, \\ Alexander Humberg ${ }^{1}$, Kathrin Hanke ${ }^{1}$, Kirstin Faust ${ }^{1}$, Julia Pagel ${ }^{1,2}$, Leila Eyvazzadeh ${ }^{1}$, \\ Kim Brenner ${ }^{1}$, Claudia Roll ${ }^{3}$, Sabine Pirr ${ }^{4}$, Dorothee Viemann ${ }^{4}$, Dimitra Stavropoulou ${ }^{5}$, \\ Philipp Henneke ${ }^{5,6}$, Birte Tröger ${ }^{7}$, Thorsten Körner ${ }^{7}$, Anja Stein ${ }^{8}$, Christoph Derouet ${ }^{9}$, \\ Michael Zemlin ${ }^{9}\left(\mathbb{D}\right.$, Christian Wieg ${ }^{10}$, Jan Rupp ${ }^{2,11}$, Egbert Herting ${ }^{1}$, Wolfgang Göpel ${ }^{1}$ and \\ Christoph Härtel ${ }^{1,2}$
}

1 Department of Pediatrics, University of Lübeck, 23562 Lübeck, Germany; janina.marissen@uksh.de (J.M.); bastian.siller@uksh.de (B.S.); uni@dr-spiegler.de (J.S.); alexander.humberg@uksh.de (A.H.); kathrin.hanke@uksh.de (K.H.); kirstin.faust@uksh.de (K.F.); julia.pagel@uksh.de (J.P.); leila.eyvazzadeh@gmail.com (L.E.); kim.brenner@uksh.de (K.B.); Egbert.Herting@uksh.de (E.H.); wolfgang.goepel@uksh.de (W.G.); christoph.haertel@uksh.de (C.H.)

2 German Center for Infection Research (DZIF), Partner Site Hamburg-Lübeck-Borstel-Riems, 38124 Braunschweig, Germany; jan.rupp@uksh.de

3 Department of Pediatrics, Vestische Children's Hospital Datteln, 45711 Datteln, Germany; c.roll@kinderklinik-datteln.de

4 Department of Neonatology, Hannover Medical School, 30159 Hannover, Germany; pirr.sabine@mh-hannover.de (S.P.); viemann.dorothee@mh-hannover.de (D.V.)

5 Center for Pediatrics and Adolescent Medicine, Medical Center and Medical Faculty, University of Freiburg, 79098 Freiburg, Germany; dimitra.stavropoulou@uniklinik-freiburg.de (D.S.); philipp.henneke@uniklinikum-freiburg.de (P.H.)

6 Institute for Immunodeficiency, Medical Center and Medical Faculty, University of Freiburg, 79098 Freiburg, Germany

7 Children's Hospital Links der Weser Bremen, 28277 Bremen, Germany; birte.troeger@gesundheitnord.de (B.T.); thorsten.koerner@klinikum-bremen-ldw.de (T.K.)

8 Department of Neonatology and General Pediatrics, University of Essen, 45147 Essen, Germany; anja.stein@uk-essen.de

9 Department of Neonatology and General Pediatrics, Saar University of Homburg, 66424 Homburg, Germany; christoph.derouet@uks.eu (C.D.); michael.zemlin@uks.eu (M.Z.)

10 Children's Hospital Aschaffenburg-Alzenau, 63739 Aschaffenburg, Germany; christian.wieg@klinikum-ab-alz.de

11 Department of Infectious Diseases and Medical Microbiology, University of Lübeck, 23562 Lübeck, Germany

* Correspondence: Matsingmar.Fortmann@uksh.de; Tel.: + 49-451-500-77328; Fax: + 49-451-500-42804

Received: 25 February 2020; Accepted: 20 March 2020; Published: 22 March 2020

Abstract: Objective: To evaluate the nutrition-related effects of prophylactic Lactobacillus acidophilus/ Bifidobacterium infantis probiotics on the outcomes of preterm infants $<29$ weeks of gestation that receive human milk and/or formula nutrition. We hypothesize that human-milk-fed infants benefit from probiotics in terms of sepsis prevention and growth. Methods: We performed an observational study of the German Neonatal Network (GNN) over a period of six years, between 1 January, 2013 and 31 December, 2018. Prophylactic probiotic use of L. acidophilus/B. infantis was evaluated in preterm infants $<29$ weeks of gestation $(n=7516)$ in subgroups stratified to feeding type: (I) Exclusively human milk (HM) of own mother and/or donors (HM group, $n=1568$ ), (II) HM of own mother and/or donor and formula (Mix group, $n=5221$ ), and (III) exclusive exposure to formula (F group, $n=727$ ). The effect of probiotics on general outcomes and growth was tested in univariate models and adjusted in linear/logistic regression models. Results: 5954 (76.5\%) infants received L. acidophilus/B. infantis 
prophylactically for the prevention of necrotizing enterocolitis (NEC). Probiotic use was associated with improved growth measures in the HM group (e.g., weight gain velocity in g/day: effect size $\mathrm{B}=0.224 ; 95 \%$ CI: 2.82-4.35; $p<0.001$ ) but not in the F group (effect size B $=-0.06$; 95\% CI: $-3.05-0.28$; $p=0.103)$. The HM group had the lowest incidence of clinical sepsis $(34.0 \%)$ as compared to the Mix group (35.5\%) and the F group (40.0\%). Only in the Mix group, probiotic supplementation proved to be protective against clinical sepsis (OR 0.69; 95\% CI: 0.59-0.79; $p<0.001$ ). Conclusion: Our observational data indicate that the exposure to L. acidophilus/B. infantis probiotics may promote growth in exclusively HM-fed infants as compared to formula-fed infants. To exert a sepsis-preventive effect, probiotics seem to require human milk.

Keywords: probiotic prophylaxis; human milk; prematurity; sepsis; growth failure

\section{Introduction}

Probiotics that act as gut colonizers of human-milk-fed infants [1] have a high potential to foster the early microbiome development [2]. Thus, they might prevent dysbiosis-associated complications such as necrotizing enterocolitis (NEC) and sepsis [3,4]. Numerous studies on the therapeutic effects of probiotics in preterm infants have been performed [4]. However, the results remain inconclusive due to a high variability in study protocols, target populations and endpoints, probiotic formulations (e.g., strain composition and inclusion of single vs. multiple strains), and the context of nutrition [5]. After the publication of several meta-analyses proposing a benefit for preterm infants' short-term outcomes, prophylaxis with Bifidobacterium longum and Bifidobacterium infantis/Lactobacillus acidophilus has been adopted into clinical routine by many European neonatal intensive care units (NICUs), for example in Austria [6], the Netherlands [7], and Germany [8]. In hospitals of the German Neonatal Network (GNN), the implementation of probiotic use in 2009/2010 led to a decrease in NEC incidence in infants discharged in 2011 and 2012 after the change of strategy [8]. Despite the use of probiotics in $>80 \%$ of extremely low-birth-weight infants (ELBWI), however, NEC and sepsis still remain significant causes of morbidity and mortality in this vulnerable population [9]. To promote a more personalized medical approach to preterm babies, there is an urgent need to define those populations who would benefit most from probiotic prophylaxis. Recent data suggest that the type of enteral feeding (breastmilk or bovine-based formula) modifies the effects of probiotics in preterm infants [6,7]. In line with this, the nutritional content of human milk oligosaccharides (HMOs) - a major metabolic source for bifidobacteria-was found to be predictive for the NEC risk [10]. Human milk contains numerous immune-related compounds such as leukocytes, lysozymes, nucleotides, and cytokines [11], whereas HMOs were found to directly mediate the prebiotic effect of bifidobacterial growth [12].

Herein we hypothesize that human milk as a nutritional source is required for probiotics to provide a sepsis-preventive and growth-promoting effect. Accordingly, we performed an observational study in a large GNN cohort of extremely preterm infants $<29$ weeks of gestation discharged after the year 2012 and evaluated the impact of probiotics in the context of feeding strategies.

\section{Methods}

\subsection{The German Neonatal Network}

The German Neonatal Network (GNN; www.vlbw.de) is a population-based observational multicenter cohort study enrolling Very low birth weight infants (VLBWI) at 64 neonatal intensive care units (NICUs) in Germany. Within the study period, data were collected from infants discharged between 1 January, 2013 and 31 December, 2018. Preterm infants of a birth weight $<1500 \mathrm{~g}$ and/or a gestational age between $22+0$ and $28+6$ weeks, who were actively managed with intensive care, met the inclusion criteria. Infants with lethal malformations or those treated with comfort 
(palliative) care were excluded from the study. In the analysis of this study, only cases with complete documentation for feeding type were included.

After obtaining written informed parental consent, predefined data on general neonatal characteristics and antenatal and postnatal treatment and outcome were recorded for each patient on clinical record files at the participating centers. After discharge, data sheets were sent to the study center (University of Lübeck). Data quality was evaluated by a physician trained in neonatology via annual on-site monitoring of completed record files. After monitoring, data were coded and evaluated.

\subsection{Prophylactic Probiotic Supplementation}

The probiotic formulation consisting of B. infantis. and L. acidophilus corresponds to the formulation that has been most commonly used among the participating study sites in the past [8]. Probiotics were provided once or twice daily in capsules beginning from day 1 to 3 of life until day 28 of life. The recommended daily dose contained $1-3 \times 10^{9} \mathrm{CFU}$ (Colony forming units) L. acidophilus and $1-1.5 \times 10^{9}$ B. infantis.

\subsection{Subgroups Stratified to Type of Milk Feeding}

The preparation (pasteurization, freezing, storage) of human milk (own mother's and donor's milk) before use was carried out according to local standards (e.g., cytomegalovirus sero-prevalence of mother) at the study site. In all centers providing donor milk, the samples were pasteurized before use for feeding. Three "human milk feeding" strata were applied:

I HM (HM group): Infants who were exclusively fed with own mother's and/or donor's milk.

II Mix (Mix group): Infants who were fed with HM and formula at any time during the primary stay in hospital.

III Formula (F group): Infants who were exclusively fed with formula.

\subsection{Definitions}

Gestational age was calculated from the "best obstetric estimate". This is defined as the estimate of the infant's gestation based on the birth attendant's final estimate by using early prenatal ultrasound and obstetric examination [13].

Small-for-gestational age (SGA) was defined as a birth weight less than the 10th percentile for gestational age according to gender-specific standards for birth weight by gestational age in Germany [14].

Full enteral feeding was defined as enteral nutrition at a minimum of $150 \mathrm{~mL} / \mathrm{kg}$ body weight per day.

Weight gain velocity was defined as gain in body weight, calculated as $\mathrm{g} /$ day (difference of the parameter at birth and at discharge/number of days in hospital). Growth velocity of body length was defined as gain in body length, calculated as $\mathrm{mm}$ /day (difference of the parameter at birth and at discharge/number of days in hospital). Head growth velocity was defined as gain in head circumference, calculated as $\mathrm{mm} /$ day (difference of the parameter at birth and at discharge/number of days in hospital). Z-scores are numerical measurements of the value's relationship to the mean of the group values measured in terms of standard deviations from the mean (between -3.0 and 3.0). Z-scores were calculated for birth weight according to the 2003 Fenton preterm growth chart $[15,16]$.

Clinical sepsis was defined as condition with at least two signs of systemic inflammatory response (temperature $>38^{\circ} \mathrm{C}$ or $<36.5^{\circ} \mathrm{C}$, tachycardia $>200 / \mathrm{min}$, new onset or increased frequency of bradycardias or apneas, hyperglycemia $>140 \mathrm{mg} / \mathrm{dL}$, base excess $<-10 \mathrm{mval} / \mathrm{L}$, changed skin color, increased oxygen requirements), one laboratory sign (e.g., C-reactive protein $>20 \mathrm{mg} / \mathrm{L}$, immature/total neutrophil ratio $>0.2$, white blood cell count $<5 / \mathrm{nL}$ ), and the neonatologist's decision to treat with anti-infective drugs for at least 5 days but no proof of causative agent in blood culture [17]. Blood culture confirmed sepsis was defined as clinical sepsis with proof of causative agent in the blood culture. 
If coagulase-negative Staphylococcus (CoNS) was isolated as a single pathogen in one peripheral blood culture, two clinical signs and one laboratory sign were required for classification of CoNS sepsis [17].

Bronchopulmonary dysplasia (BPD) was diagnosed when needing supplemental oxygen or ventilatory support at 36 weeks of postmenstrual age. Necrotizing enterocolitis (NEC) was defined as necrotizing intestinal inflammation requiring surgery, and focal intestinal perforation (FIP) was FIP requiring surgical treatment classified by the attending surgeon. Retinopathy of prematurity (ROP) was defined as typical retinal changes (ophthalmoscopy) requiring interventions such as laser therapy, cryotherapy, or intraocular vascular endothelial growth factor inhibitors.

\subsection{Statistical Analyses}

Data analyses were performed using the SPSS 24.0 data analysis package (Munich, Germany). Hypotheses in the univariate analysis were evaluated with Fisher's exact test and Mann-Whitney U test. Only two-sided tests were used. A $p$-value of $<0.05$ was considered as statistically significant for single tests.

Subsequent to univariate analyses, we included parameters with a $p$-value $<0.1$ in multivariate logistic regression models and known confounders as independent variables: gestational age per week, gender, multiples, and SGA status. Odd ratios and 95\% confidence intervals were calculated in order to identify the influence of probiotic prophylaxis on outcomes independent of the abovementioned confounders. The following outcome parameters were tested in multivariate models: necrotizing enterocolitis, retinopathy of prematurity requiring intervention, bronchopulmonary dysplasia, clinical sepsis, and blood culture confirmed sepsis. To address the problem of multiple comparisons, we performed Bonferroni corrections for multivariate analyses in order to protect from statistical Type I errors. Additional information derived from Bonferroni correction is indicated in Tables 1 and 2 accordingly. In addition, we tested nested models for our analyses by calculating the Akaike information criterion (AIC) for all multivariate calculations in order to estimate relative quality for each model.

Table 1. Effect of probiotic treatment on short-term outcomes in the context of feeding types.

\begin{tabular}{cccc}
\hline & I HM & II Mix & III Formula \\
\hline \multirow{2}{*}{ Surgery for NEC } & OR 1.37 (95\% CI: 0.69-2.73) & OR 0.84 (95\% CI: 0.59-1.15) & OR 0.89 (95\% CI: 0.5-2.0) \\
& $p=0.38$ & $p=0.26$ & $p=0.9$ \\
Clinical sepsis & OR 0.95 (95\% CI: 0.73-1.22) & OR 0.69 (95\% CI: $0.59-0.79)$ & OR 1.20 (95\% CI: 0.9-1.7) \\
& $p=0.67$ & $p<0.001 *$ & $p=0.243$ \\
Sepsis (BC positive) & OR 1.09 (95\% CI: 0.78-1.53) & OR 0.89 (95\% CI: 0.74-1.06) & 1.10 (95\% CI: 0.7-1.8) \\
& $p=0.60$ & $p=0.19$ & $p=0.662$ \\
ROP & OR 1.51 (95\% CI: 0.73-3.10) & OR $1.04(95 \%$ CI: $0.72-1.51)$ & OR 1.35 (95\% CI: \\
& $p=0.27$ & $p=0.83$ & $0.63-2.94)$ \\
BPD & OR 0.86 (95\% CI: 0.65-1.14) & OR 0.90 (95\% CI: $0.77-1.05)$ & $1.31(95 \%$ CI: $0.87-1.96)$ \\
& $p=0.31$ & $p=0.19$ & $p=0.19$ \\
\hline
\end{tabular}

HM, human milk, NEC, necrotizing enterocolitis, ROP, retinopathy of prematurity, BPD, bronchopulmonary dysplasia; NEC, necrotizing enterocolitis. Logistic regression analyses were performed by using the following independent variables: gestational age, multiple birth, gender, SGA, and treatment with probiotics. * Bonferroni correction did not change significance of the $p$-value. 
Table 2. Effect of probiotics on growth parameters of the GNN cohort during their primary stay in hospital.

\begin{tabular}{|c|c|c|c|}
\hline & I HM & II Mix & III Formula \\
\hline Body weight a & $B=0.261$ & $B=0.026$ & $B=0.015$ \\
\hline$(\mathrm{z}-\mathrm{sco}$ & $95 \%$ CI: $0.48-0.71 p<0.001 *$ & $95 \%$ CI: $0.01-0.1 p=0.029 \#$ & $95 \%$ CI: $-0.12-0.18 p=0.72$ \\
\hline gain & $B=0.23$ & $\mathrm{~B}=0.022$ & $\mathrm{~B}=0.06$ \\
\hline (z-score, Fenton) & $95 \%$ CI: $0.42-0.62 p<0.001$ * & $95 \%$ CI: $-0.01-0.1 p=0.078$ & $95 \%$ CI: $-0.04-0.26 p=0.14$ \\
\hline & $B=0.224$ & $\mathrm{~B}=0.00$ & $B=-0.06$ \\
\hline Grow & $95 \%$ CI: $2.82-4.35 p<0.001 *$ & $95 \%$ CI: $-0.61-0.62 p=0.98$ & $95 \%$ CI: $-2.90--0.45 p=0.15$ \\
\hline Grow & $B=0.179$ & $B=0.019$ & $B=-0.012$ \\
\hline le & 95\% CI: $0.13-0.24 p<0.001$ * & $95 \%$ CI: $-0.01-0.04 p=0.184$ & $95 \%$ CI: $-0.11-0.08 p=0.761$ \\
\hline Head growth velocity & $B=0.117$ & $B=0.03$ & $B=-0.002$ \\
\hline (mm/day) & $95 \%$ CI: $0.05-0.12 p<0.001^{*}$ & $95 \%$ CI: $0.003-0.04 p=0.023$ \# & $95 \%$ CI: $-0.08-0.07 p=0.966$ \\
\hline
\end{tabular}

HM, human milk. Growth velocity, weight gain, and growth of body length and head circumference were calculated by differences between parameters at birth and respective measures at discharge/number of days (duration of stay). Linear regression analyses were performed by using the following independent variables: gestational age, birth weight, multiple birth, gender, maternal descent, and exposure to probiotic prophylaxis within the three subgroups.

* Bonferroni correction did not change significance of the p-value. ${ }^{\#}$ Not significant after Bonferroni correction.

In order to evaluate the influence of probiotic prophylaxis on growth parameters, we conducted linear regression models using known/probable confounders as independent variables: gestational age per week, birth weight, gender, multiple birth, and maternal descent. Effect size and 95\% confidence intervals (CI) were calculated. The following outcome parameters were tested in linear models: Z-score-based body weight at discharge according the 2003 Fenton growth chart for preterm infants, z-score-based weight gain, growth velocity of body weight (g/day), body length (mm/day) and head circumference (mm/day). A $p$-value of $<0.05$ was considered statistically significant. For primary and subgroup analyses, we used a uniform dataset with available data for all metric parameters. Infants with incomplete data for variables that were used in our analyses were not included.

Graphical analysis was carried out using GraphPad Prism (Version 6.00, GraphPad Software, La Jolla, CA, USA).

\subsection{Ethical Approval}

All study parts were ethically approved by the University of Lübeck Ethical Committee and the committees of the participating centers (vote no. 08-022). Informed consent was obtained from all subjects. All methods were carried out in accordance with relevant guidelines and regulations, specifically: the Declaration of Helsinki, the current revision of ICH (The International Council for Harmonisation) Topic E6, the Guidelines for Good Clinical Practice, and the Guidelines of the Council for International Organization of Medical Sciences, the WHO (World Health Organization) ("Proposed International Guidelines for Biomedical Research Involving Human Subjects").

\section{Results}

From 1 January, 2013 until 31 December, 2018, 7516 extremely low gestational age neonates (ELGANs) were discharged in $64 \mathrm{GNN}$ centers. The study cohort had a mean gestational age at birth of 26.5 weeks (median 26.7 weeks; SD 1.6 weeks) and a mean birth weight of $855 \mathrm{~g}$ (median $845 \mathrm{~g}$; SD $248 \mathrm{~g}$, Table 3). Patients were hospitalized for a median of 85 days. Moreover, 1568 infants (20.9\%) were exclusively fed with human milk (HM) during their primary stay, 5221 infants $(69.5 \%)$ received both HM of mothers/donors and formula (Mix group), and 727 infants (9.6\%) were fed exclusively formula nutrition (F group). 
Table 3. Clinical characteristics of the GNN cohort stratified to type of milk feeding.

\begin{tabular}{lccccc}
\hline & I HM & II Mix & III Formula & $\begin{array}{c}p \text {-Value (HM } \\
\text { vs. Formula) }\end{array}$ & Total \\
\hline Number of infants n, (\%) & $1568,(20.9)$ & $5221,(69.5)$ & $727,(9.6)$ & & $7516,(100)$ \\
Gestational age (weeks) & $26.4 / 1.68(26.57)$ & $26.52 / 1.61(26.7)$ & $26.6 / 1.95(26.9)$ & $0.024^{\#}$ & $26.5 / 1.63(26.7)$ \\
Birth weight (g) & $841 / 257(830)$ & $861 / 245(850)$ & $858 / 243(850)$ & $0.067 \#$ & $855 / 248(845)$ \\
Z-score (birth weight) & $-0.25 / 0.98$ & $-0.20 /-0.92$ & $-0.25 /-0.16$ & $0.661^{\#}$ & $-0.22 / 0.93$ \\
Gender, male (\%) & $(-0.15)$ & $(0.13)$ & $(0.03)$ & 0.380 & $(-0.14)$ \\
Multiples (\%) & 54.4 & 52.9 & 52.4 & 0.020 & 33.2 \\
SGA (\%) & 30.9 & 34.4 & 26.1 & 0.526 & 12.9 \\
Caesarean section (\%) & 14.6 & 12.1 & 13.6 & 0.471 & 89.9 \\
Vaginal delivery (\%) & 88.4 & 90.8 & 87.3 & 0.471 & 10.1 \\
Hospitalization (days) & 11.6 & 9.2 & 12.7 & $0.372 \#$ & $85 / 40(80)$ \\
Time to full enteral feeds & $83 / 39(79)$ & $87 / 38(81)$ & $85 / 45(80)$ & $0.341^{\#}$ & $18.2 / 15.1(14.0)$ \\
(days) & $18.5 / 14.6(14.0)$ & $17.7 / 14.3(14.0)$ & $19.8 / 1(17.8)$ & & \\
Duration of intravenous & $26.0 / 23.7(18.0)$ & $26.2 / 25.1(18.0)$ & $28.1 / 27.6(18.0)$ & $0.7799^{\#}$ & $26.4 / 25.3(18.0)$ \\
line (days) & & & & & \\
\hline
\end{tabular}

HM, human milk; SGA, small-for-gestational-age (<10th Voigt percentile); $p$-values were derived from chi-square test, if not otherwise indicated ( ${ }^{\#}$, Mann-Whitney-U test). Continuous variables and $z$-scores are shown as mean/SD (median).

\subsection{Human Milk Feeding Has Increased in GNN Centers from 2013 to 2018}

To account for time trends in enteral feeding practices and current developments in human milk banks, we evaluated the proportion of infants receiving human milk (own-mother; donor milk), mix, or exclusively formula according to the year of the infant's discharge. There has been an increasing rate of ELGANs that receive HM (72.8\% in 2013 versus 91.3\% in 2018; Figure 1) during primary stay in hospital and at discharge ( $47.2 \%$ in 2013 versus $66.6 \%$ in 2018). The administration of donor milk started in 2013 in $3.1 \%$ of infants and increased to $22.7 \%$ in 2018.

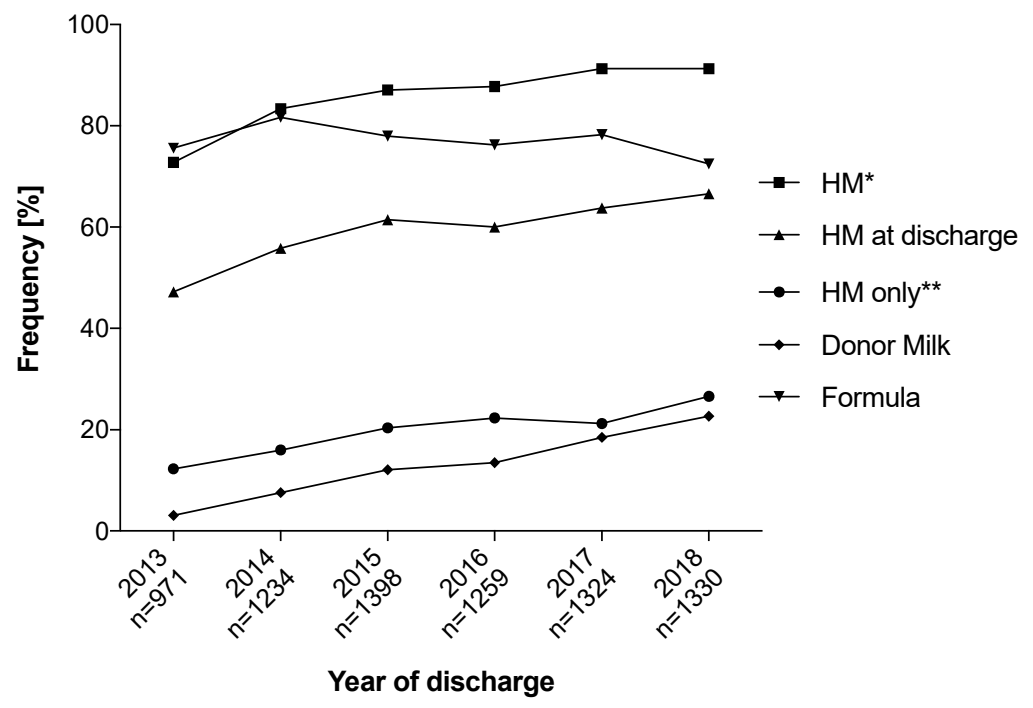

Figure 1. Changes in enteral feeding on German Neonatal Network (GNN) neonatal intensive care units (NICUs) between 2013 and 2018. HM, human milk; * includes all infants that were exposed to $\mathrm{HM}$; ${ }^{* *}$ includes all infants that were fed exclusively HM (own mother and/or donor).

\subsection{Formula-Fed Infants Have an Increased Risk for Adverse Short-Term Outcomes}

In Table 3, the clinical characteristics of the groups are described with no major differences between the groups. L. acidophilus/B. infantis probiotics were administered prophylactically to 5682 ELGAN (76.5\%) infants, with a frequency of $74.8 \%$ for infants receiving HM (HM group) and $74.9 \%$ for those with formula (F group; $p=0.935$ ). Notably univariate analyses revealed that the F group had 
significantly higher rates of NEC (6.2\% vs. 3.1\%; $p<0.001)$, clinical sepsis $(40.2 \%$ vs. $34.0 \%$; $p=0.004)$, and severe ROP $(6.5 \%$ vs. $3.2 \% ; p=0.004)$ as compared to the HM group. Exclusively formula-fed infants (F group) had a higher body weight at discharge and weight gain/day, head circumference, and body length than infants receiving HM in univariate analyses $(p<0.001$, Table 4$)$.

Table 4. Treatment, outcomes, and growth parameters of the GNN cohort stratified to type of milk feeding.

\begin{tabular}{|c|c|c|c|c|c|}
\hline & I HM & II Mix & III Formula & $\begin{array}{l}p \text {-Value (HM } \\
\text { vs. Formula) }\end{array}$ & Total \\
\hline Number of infants $n,(\%)$ & $1568(20.9)$ & $5221(69.5)$ & $727(9.6)$ & & $7516(100)$ \\
\hline Antibiotic treatment (\%) & 94.3 & 93.5 & 95.3 & 0.323 & 93.7 \\
\hline Probiotic prophylaxis (\%) & 74.8 & 78.3 & 74.9 & 0.935 & 76.5 \\
\hline Surgery for NEC (\%) & 3.1 & 3.8 & 6.2 & $<0.001^{\#}$ & 3.9 \\
\hline BC-confirmed sepsis (\%) & 14.6 & 16.8 & 15.7 & 0.506 & 16.3 \\
\hline Clinical sepsis (\%) & 34.0 & 35.5 & 40.2 & 0.004 & 35.6 \\
\hline $\mathrm{BPD}(\%)$ & 26.4 & 28.5 & 28.7 & 0.240 & 28.0 \\
\hline Severe ROP (\%) & 3.2 & 3.6 & 6.5 & 0.004 & 3.9 \\
\hline Weight $(\mathrm{g})$ at discharge & $\begin{array}{l}2460 / 750 \\
(2460)\end{array}$ & $\begin{array}{l}2711 / 733 \\
(2650)\end{array}$ & $\begin{array}{l}2752 / 1020 \\
(2731)\end{array}$ & $<0.001^{\#}$ & $\begin{array}{c}2653 / 818 \\
(2615)\end{array}$ \\
\hline Z-score (birth weight) & $\begin{array}{c}-1.73 /-1.63 \\
(0.03)\end{array}$ & $\begin{array}{c}-1.35 /-1.32 \\
(0.85)\end{array}$ & $\begin{array}{c}-1.22 /-1.22 \\
(0.79)\end{array}$ & $<0.001^{\#}$ & $\begin{array}{c}-1.40 / 0.88 \\
(-1.36)\end{array}$ \\
\hline Weight gain velocity (g/day) & $19.1 / 6.3(19.7)$ & $21.5 / 9.3(21.6)$ & $22.2 / 10.1(22.4)$ & $<0.001^{\#}$ & $21.1 / 9.09(21.4)$ \\
\hline $\begin{array}{l}\text { Weight at discharge } \\
\text { (z-scores, Fenton) }\end{array}$ & $\begin{array}{c}-1.53 / 1.42 \\
(0.03)\end{array}$ & $\begin{array}{c}-1.16 /-1.12 \\
(0.83)\end{array}$ & $\begin{array}{c}-1.03 /-0.99 \\
(0.79)\end{array}$ & $<0.001^{\#}$ & $\begin{array}{l}-1.2 / 0.86 \\
(-1.15)\end{array}$ \\
\hline $\begin{array}{l}\text { Growth velocity of body } \\
\text { length }(\mathrm{mm} / \text { day })\end{array}$ & $1.36 / 0.44(1.37)$ & $1.43 / 0.39(1.42)$ & $1.49 / 0.48(1.45)$ & $<0.001^{\#}$ & $1.42 / 0.41(1.41)$ \\
\hline $\begin{array}{l}\text { Head growth velocity } \\
\text { (mm/day) }\end{array}$ & $0.98 / 0.37(0.98)$ & $1.03 / 0.28(1.03)$ & $1.06 / 0.40(1.02)$ & $<0.001^{\#}$ & $1.02 / 0.31(1.02)$ \\
\hline
\end{tabular}

HM, human milk; BPD, bronchopulmonary dysplasia; NEC, necrotizing enterocolitis; ROP, retinopathy of prematurity; BC, blood culture. Growth velocity and weight gain were calculated by differences between parameters at birth and respective measures at discharge/number of days (duration of stay). Continuous variables and z-scores are shown as mean/SD (median); $p$-values were derived from Fisher's exact test or Mann-Whitney-U test if indicated with $\#$.

\subsection{Probiotics Reduce the Risk for Clinical Sepsis in Infants with Human Milk and Formula Exposure}

In the HM group, probiotics were not associated with reduced risk for adverse outcomes such as sepsis or BPD. In the Mix group, probiotics were associated with a risk reduction for clinical sepsis compared to untreated infants (univariate: $33.9 \%$ vs. $41.3 \%, p<0.001$; logistic regression: OR $0.69 ; 95 \%$ CI: $0.59-0.79, p<0.001$; Tables 1 and 5 ). There were no significant effects of probiotics on the risk of NEC, ROP, sepsis, and BPD in the F group.

Table 5. Outcomes of the GNN cohort stratified to type of milk feeding and treatment with probiotics.

\begin{tabular}{|c|c|c|c|c|c|c|c|c|c|}
\hline & \multicolumn{2}{|c|}{ I HM } & \multirow{2}{*}{$p$} & \multicolumn{2}{|c|}{ II Mix } & \multirow{2}{*}{$p$} & \multicolumn{2}{|c|}{ III Formula } & \multirow{2}{*}{$p$} \\
\hline & $\begin{array}{c}\text { No } \\
\text { Probiotics }\end{array}$ & Probiotics & & $\begin{array}{c}\text { No } \\
\text { Probiotics }\end{array}$ & Probiotics & & $\begin{array}{c}\text { No } \\
\text { Probiotics }\end{array}$ & Probiotics & \\
\hline Number of infants $n,(\%)$ & $\begin{array}{c}395 \\
(25.2)\end{array}$ & $\begin{array}{l}1173 \\
(74.8)\end{array}$ & & $\begin{array}{l}1135 \\
(21.7)\end{array}$ & $\begin{array}{c}4086 \\
(78.3)\end{array}$ & & $\begin{array}{c}182 \\
(25.1)\end{array}$ & $\begin{array}{c}545 \\
(74.9)\end{array}$ & \\
\hline $\operatorname{NEC}(\%)$ & 2.8 & 3.3 & 0.65 & 4.4 & 3.6 & 0.21 & 7.2 & 5.9 & 0.54 \\
\hline Clinical sepsis (\%) & 36.6 & 33.2 & 0.02 & 41.3 & 33.9 & $<0.001^{\#}$ & 37.0 & 41.0 & 0.35 \\
\hline BC-confirmed sepsis (\%) & 14.6 & 14.6 & 0.98 & 18.2 & 16.5 & 0.17 & 15.0 & 15.8 & 0.79 \\
\hline Severe ROP (\%) & 2.7 & 3.5 & 0.45 & 3.8 & 3.6 & 0.76 & 2.9 & 7.4 & 0.34 \\
\hline $\mathrm{BPD}$ & 30.5 & 25.0 & 0.003 & 29.5 & 28.2 & 0.38 & 26.5 & 29.3 & 0.48 \\
\hline
\end{tabular}

HM, human milk; BPD, bronchopulmonary dysplasia; NEC, necrotizing enterocolitis; ROP, retinopathy of prematurity; BC, blood culture. Continuous variables and $z$-scores are shown as mean/SD (median). Categorical variables are shown as percent. $p$-values were derived from Fisher's exact test or Mann-Whitney-U test if indicated with ${ }^{\#}$. 


\subsection{Probiotics Have a Growth-Promoting Effect in Exclusively Human-Milk-Fed Infants}

A major effect of probiotic administration on growth was only observed in the HM group, i.e., higher body weight at discharge including Fenton $z$-scores $(-1.49$ vs. $-2.13 ; p<0.001)$, $z$-score-based weight gain $(-1.30$ vs. $-1.83 ; p<0.001)$, and growth rates of body weight, body length, and head circumference (Table 6). Weight gain velocity of HM infants (20.7 g/day) almost reached levels of $\operatorname{mix}(21.5 \mathrm{~g} /$ day), and formula-fed infants $(21.9 \mathrm{~g} /$ day $)$. In order to address confounding factors such as gestational age (catch-up growth of smaller infants), we performed a linear regression model including gestational age, birth weight, gender, multiple birth, and maternal descent (Table 2). In HM infants, probiotics were associated with higher bodyweight at discharge (effect size $B=0.261 ; 95 \%$ CI: $0.48-0.71 ; p<0.001)$ and $z$-score-based weight gain. Notably probiotic treatment was associated with increased weight gain velocity (effect size B $=0.224 ; 95 \% \mathrm{CI}: 2.82-4.35 ; p<0.001$ ), body length, and head circumference. This (probiotic) effect was observed to a lesser extent in the Mix group (body weight at discharge, head growth velocity), while Formula infants were not affected by probiotics in that aspect (Table 2).

Table 6. Growth parameters of the GNN cohort stratified to type of milk feeding and treatment with probiotics.

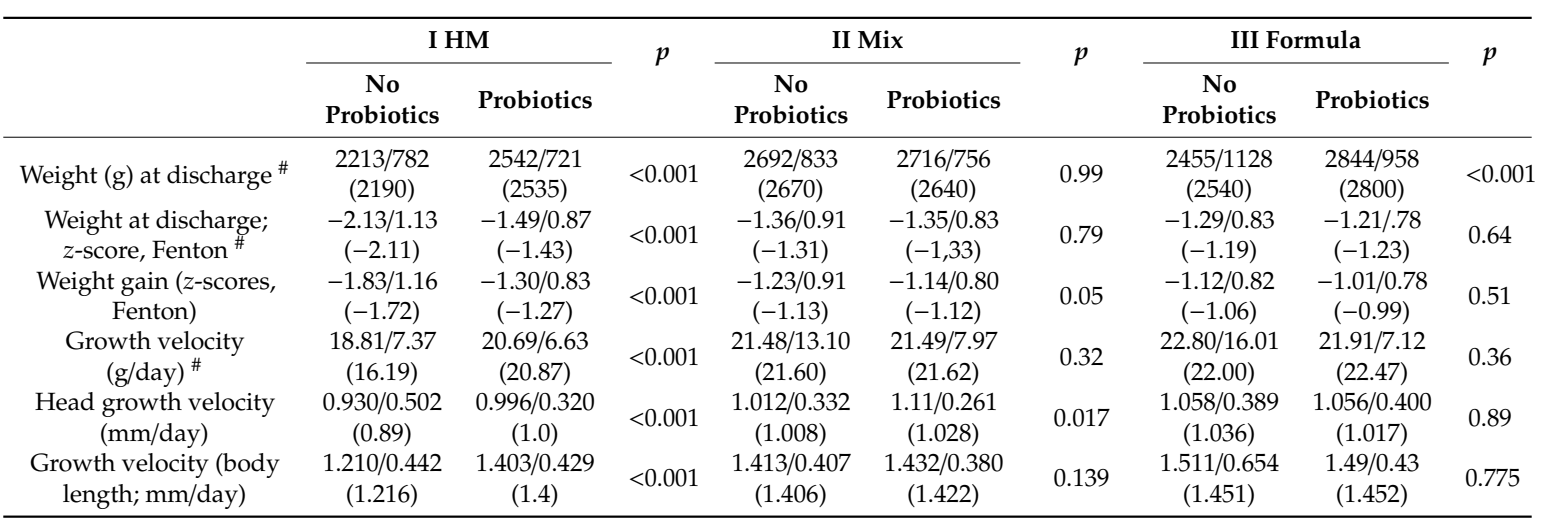

HM, human milk. Growth velocity, weight gain, and growth of body length and head circumference were calculated by differences between parameters at birth and respective measures at discharge/number of days (duration of stay). Continuous variables and z-scores are shown as mean/SD (median), $p$-values were derived from Mann-Whitney-U test if indicated with ${ }^{\#}$.

\section{Discussion}

Our large-scale population-based data support the hypothesis that the source of enteral feeding has an impact on the effects of L. acidophilus/B. infantis probiotics in highly vulnerable preterm infants. In this context, human milk exposure is required for probiotics to provide a sepsis-preventive and growth-promoting effect. Exclusively formula-fed infants did not benefit from the administration of probiotics in terms of sepsis, NEC, or BPD risk.

The feeding of preterm infants with human milk has been previously associated with reduced morbidity and mortality [18-21]. The stabilization of the early host-microbiome interaction has been proposed as a crucial underlying mechanism for this beneficial effect [12]. On the other hand, human milk might not fully meet the nutritional requirements of preterm infants [22], which results in less weight gain in human-milk-fed preterm babies as compared to formula-fed infants [23]. Bovine multicomponent fortification of human milk has been proposed to cause inflammation [24], and there is a lack of scientific evidence about whether or not its routine use can impact growth and other short- and long-term outcomes [25]. Furthermore, there is uncertainty on whether pasteurized donated human milk should be preferred to preterm formula [26]. In clinical reality, most babies in participating GNN units receive a mix of human milk and formula while donor human milk availabilities are increasingly being established but still limited. We noted a temporal trend to higher rates of human milk feeding, which approached $90 \%$ in 2018. In such a setting with low NEC rates $<4 \%$ in babies $<29$ weeks and $75 \%$ 
exposure to probiotics, we expected no further risk reduction for NEC by probiotics as compared to our previous findings before/after the introduction of probiotics into clinical routine [8]. In the current cohort study, we noted a promoting effect of probiotics on weight gain and growth velocities in HM-fed infants. Previous studies revealed inconsistent results that were not necessarily adjusted for the type of feeding [27-30]. A meta-analysis of 15 studies including 3751 infants $<32$ weeks and $<1500 \mathrm{~g}$ birth weight demonstrated no significant effects of probiotics on weight gain [31]. In comparison to our cohort, the gestational age in this pooled study cohort analysis was higher while mean weight gain/day was lower (16 g/day vs. $21 \mathrm{~g} /$ day) indicating that the effects of probiotics are context sensitive. While the huge variability in study designs has been acknowledged, the authors conclude that probiotics are more effective in reducing morbidity when taken in human milk or human milk plus formula form, consumed for $<6$ weeks, administered at a dosage of $<10^{9} \mathrm{CFU} / \mathrm{d}$, and include multiple strains. We assume that promoted weight gain in HM-fed infants is correlated with the dynamics of the establishing intestinal microbiota, nutrient utilization, and immune-metabolome interaction [4,32]. L. acidophilus/B. infantis may require the complex composition of human milk to exert a growth-promoting effect and to stabilize gut immunity in order to prevent translocation sepsis $[6,7,33]$. Human milk includes endogenous probiotics, prebiotic carbohydrates, stem cells, and a concert of bioactive human milk factors (e.g., S100 A8/9 [34]) that have direct or indirect effects on the vulnerable host-gut microbiota interplay [11]. Hence, the sepsis-preventive effect of human milk may not be additionally increased by probiotics in exclusively HM-fed infants. Infants who are exposed to human milk and formula benefit from probiotics, which would compensate for the reduced abundance of bifidobacteria and lactobacilli in their gut microbiota composition [20,35]. Both, human milk feeding and probiotics might also be able to "reverse" antibiotic-induced gut dysbiosis [36], which needs to be subject to further long-term studies of extremely preterm infants.

\section{Strengths and Limitations}

The major strengths of our study design are the large sample size and high quality of the clinical data that are monitored on-site by a study team trained in neonatology. The main limitations are the observational design, the lack of information on the daily type of feeding in the Mix group, indication for supplementation, and timing with bovine and individual fortification of human milk or formula. Furthermore, we did not have exact data on the timing of probiotics, the number of pasteurized milk portions, and the bacterial load of human own-mother milk (if not pasteurized) in the individual infants. Whether probiotics or the probiotic/prebiotic load of human milk is causal for the observed effects, needs to be addressed in future studies including extensive sequencing of human milk microbiome and the gut microbiome of the milk (+probiotic)-fed infants.

\section{Conclusions}

Evidence of nutrition-related effects of probiotic prophylaxis in preterm infants is scarce. In a large cohort of VLBWI, we conclude that supplementation of L. acidophilus/B. infantis and feeding strategies interact and have the potential of improving outcomes and growth in preterm infants. Our data demonstrates sepsis-preventive and growth-promoting effects exclusively in infants receiving human milk, supporting usage of human milk (including human milk from donors) in preterm infants whenever possible. Randomized, placebo-controlled trials as the PRIMAL clinical study [37] are pending to test hypothesis generating observational studies and to evaluate long-term effects of probiotics. 
Author Contributions: Conceptualization, I.F., E.H., W.G., and C.H.; data curation, I.F., C.R., P.H., W.G., and C.H.; formal analysis, I.F., J.M., B.S., J.S., A.H., K.H., K.F., J.P., L.E., K.B., C.R., S.P., D.V., D.S., P.H., B.T., T.K., A.S., C.D., M.Z., C.W., J.R., E.H., W.G., and C.H.; funding acquisition, E.H., W.G., and C.H.; investigation, I.F., J.M., C.R., S.P., D.V., P.H., B.T., T.K., A.S., E.H., W.G., and C.H.; methodology, I.F., J.S., K.H., C.R., P.H., A.S., E.H., W.G., and C.H.; project administration, W.G. and C.H.; resources, E.H., W.G., and C.H.; supervision, J.S., E.H., W.G., and C.H.; visualization, I.F.; writing - original draft, I.F. and C.H.; writing—review and editing, J.M., B.S., J.S., A.H., K.H., K.F., J.P., L.E., K.B., C.R., S.P., D.V., D.S., P.H., B.T., T.K., A.S., C.D., M.Z., C.W., J.R., E.H., and W.G. All authors have read and agreed to the published version of the manuscript.

Acknowledgments: The authors are indebted to all parents and their infants. We thank all nurses and doctors and study centers who participate in the GNN. The participants of the German Neonatal Network (GNN) are: Wolfgang Göpel, Department of Pediatrics, University of Lübeck; Meike Bendiks, Children's Hospital, University of Kiel; Martin Andree Berghäuser, Department of Pediatrics, Florence Nightingale Hospital Düsseldorf; Corinna Gebauer, Department of Pediatrics, University of Leipzig, Kai Bockenholt, Children's Hospital Cologne, Amsterdamer Strasse; Ralf Boettger, Perinatal Centre, University of Magdeburg; Bettina Bohnhorst, Department of Neonatology, Hannover Medical School; Thomas Brune, Children's Hospital Lippe; Kristin Dawczynski, Department of Neonatology, University of Jena; Michael Dördelmann, Diakonissenhospital Flensburg; Silke Ehlers, Bürgerhospital Frankfurt; Joachim G. Eichhorn, Children's Hospital Leverkusen; Ursula-Felderhoff-Müser, Department of Pediatrics, University of Essen; Axel Franz, Department of Neonatology, University of Tübingen; Hubert Gerleve, Children's Hospital Coesfeld; Ludwig Gortner, Department of Pediatrics, University of Homburg; Florian Guthmann, Children's Hospital Auf der Bult, Hannover; Roland Haase, Department of Pediatrics, University of Halle; Friedhelm Heitmann, Children's Hospital Dortmund; Michael Heldmann, Department of Neonatology, Helios Hospital Wuppertal; Nico Hepping, St. Marienhospital, Bonn; Roland Hentschel, Department of Pediatrics and Neonatology, University of Freiburg; Georg Hillebrand, MD, Children's Hospital Itzehoe; Thomas Hoehn, Department of Pediatrics, University of Düsseldorf; Mechthild Hubert, Children's Hospital Siegen; Helmut Hummler, Department of Neonatology, University of Ulm; Reinhard Jensen, Children's Hospital Heide; Olaf Kannt, Children's Hospital Schwerin; Thorsten Koerner, Department of Neonatology, Hospital Links der Weser, Bremen; Helmut Küster, Department of Pediatrics, University of Göttingen; Angela Kribs, Department of Pediatrics, University of Cologne, Knud Linnemann, Department of Pediatrics, Ernst-Moritz-Arndt-University of Greifswald; Jens Möller, Children's Hospital Saarbrücken; Dirk Mueller, Children's Hospital Kassel; Dirk Olbertz, Department of Neonatology, Hospital Suedstadt Rostock; Thorsten Orlikowsky, Department of Neonatology, University of Aachen; Jochen Reese, Children's Hospital Eutin; Claudia Roll, Department of Pediatrics, University Witten-Herdecke, Vestische Children's Hospital Datteln; Rainer Rossi, Children's Hospital Berlin-Neukoelln, Berlin; Mario Rüdiger, Department of Neonatology, University of Dresden; Stefan Schaefer, Department of Neonatology, Hospital Nürnberg Süd; Thomas Schaible, Department of Pediatrics, University of Mannheim; Susanne Schmidtke, Department of Neonatology, Asklepios Hospital Hamburg-Barmbek; Stefan Seeliger, Children's Hospital Neuburg/ Ingolstadt; Hugo Segerer, Children's Hospital St. Hedwig, Regensburg; Norbert Teig, Department of Pediatrics, University of Bochum; Hans-Georg Topf, Department of Neonatology, University of Erlangen; Florian Urlichs, Department of Pediatrics, St. Franziskus-Hospital Muenster; Matthias Vochem, Olgahospital Stuttgart; Ursula Weller, Children's Hospital Bielefeld; Axel von der Wense, Children's Hospital Altona e.V.; Claudius Werner, Children's Hospital, Westfälische Wilhelms-Universitiy of Muenster; Christian Wieg, Children's Hospital Aschaffenburg-Alzenau; Jürgen Wintgens, Children's Hospital Mönchengladbach; Welfhard Schneider, Vivantes Hospital Friedrichshain; Esther Rieger-Fackeldey, Department of Neonatology, Klinikum rechts der Isar, Technical University, Munich; Friedrich Ebinger, St. Vincent Hospital Paderborn; Gernot Sinnecker, Department of Pediatrics Wolfsburg; Volkmar Kunde, Christian Children's Hospital Osnabrück and Thomas Völkl, Department of Pediatrics Josefinum Augsburg. We thank Anja Kaufmann, Birgit Schröder, Tim Röntgendorf, Liane Triebwasser, and Sabine Lorenz for data documentation. The participants of the PRIMAL Consortium are Philipp Henneke, Center for Pediatrics and Adolescent Medicine and Institute for Immunodeficiency, University of Freiburg, Germany; Christoph Härtel, Department of Pediatrics, University of Lübeck, Germany; Peer Bork, Structural and Computational Biology Unit, European Molecular Biology Laboratory, Heidelberg, Germany; Stephan Gehring, Department of Pediatrics, University Medical Centre, Mainz, Germany; David Frommhold, Children's Hospital Memmingen, Memmingen, Germany; Michael Zemlin, Department of General Pediatrics and Neonatology, Saarland University, Homburg, Germany; Christian Gille, Department of Neonatology, University of Tübingen, Tübingen, Germany; Dorothee Viemann, Department of Pediatric Pneumology, Allergology, and Neonatology, Hannover Medical School, Hannover, Germany.

Conflicts of Interest: The authors declare no conflict of interests. GNN is funded by the German Ministry for Education and Research. There has been no involvement in study design, collection of analysis, interpretation of data, writing of the report, and decision to submit the manuscript for publication by the German Ministry for Education and Research. The first version of the manuscript was written by Ingmar Fortmann and Christoph Härtel. No payment, honorarium, grant, or other form of payment has been given to the authors. 


\section{Abbreviations}

$\begin{array}{ll}\text { BPD } & \text { bronchopulmonary dysplasia } \\ \text { HM } & \text { human milk } \\ \text { ELGAN } & \text { extremely low gestational age neonates } \\ \text { FIP } & \text { focal intestinal perforation } \\ \text { GNN } & \text { German Neonatal Network } \\ \text { NEC } & \text { necrotizing enterocolitis } \\ \text { NICU } & \text { neonatal intensive care unit } \\ \text { ROP } & \text { retinopathy of prematurity } \\ \text { SGA } & \text { small for gestational age }\end{array}$

\section{References}

1. Underwood, M.A.; Kalanetra, K.M.; Bokulich, N.A.; Lewis, Z.T.; Mirmiran, M.; Tancredi, D.J.; Mills, D.A. A comparison of two probiotic strains of bifidobacteria in premature infants. J. Pediatr. 2013, 163, 1585-1591. [CrossRef]

2. Warner, B.B.; Tarr, P.I. Necrotizing enterocolitis and preterm infant gut bacteria. Semin. Fetal Neonatal Med. 2016, 21, 394-399. [CrossRef]

3. Olsen, R.; Greisen, G.; Schrøder, M.; Brok, J. Prophylactic probiotics for preterm infants: A systematic review and meta-analysis of observational studies. Neonatology 2016, 109, 105-112. [CrossRef]

4. Graspeuntner, S.; Waschina, S.; Kunzel, S.; Twisselmann, N.; Rausch, T.K.; Cloppenborg-Schmidt, K.; Viemann, D.; Herting, E.; Göpel, W.; Baines, J.F. Gut dysbiosis with Bacilli dominance and accumulation of fermentation products precedes late-onset sepsis in preterm infants. Clin. Infect. Dis. 2019, 69, 268-277. [CrossRef]

5. Costeloe, K.; Hardy, P.; Juszczak, E.; Wilks, M.; Millar, M.R. Study PPI. Bifidobacterium breve BBG-001 in very preterm infants: A randomised controlled phase 3 trial. Lancet 2016, 387, 649-660. [CrossRef]

6. Repa, A.; Thanhaeuser, M.; Endress, D.; Weber, M.; Kreissl, A.; Binder, C.; Berger, A.; Haiden, N. Probiotics (Lactobacillus acidophilus and Bifidobacterium infantis) prevent NEC in VLBW infants fed breast milk but not formula. Pediatr. Res. 2015, 77, 381-388. [CrossRef]

7. Samuels, N.; van de Graaf, R.; Been, J.V.; de Jonge, R.C.J.; Hanff, L.M.; Wijnen, R.M.H.; Kornelisse, R.F.; Reiss, I.K.M.; Vermeulen, M.J. Necrotising enterocolitis and mortality in preterm infants after introduction of probiotics: A quasi-experimental study. Sci. Rep. 2016, 6, 31643. [CrossRef]

8. Härtel, C.; Pagel, J.; Rupp, J.; Bendiks, M.; Guthmann, F.; Rieger-Fackeldey, E.; Heckmann, M.; Franz, A.; Schiffmann, J.H.; Zimmermann, B. Prophylactic Use of Lactobacillus acidophilus/Bifidobacterium infantis Probiotics and Outcome in Very Low Birth Weight Infants. J. Pediatr. 2014, 165, 285-289.e1. [CrossRef] [PubMed]

9. Humberg, A.; Härtel, C.; Rausch, T.K.; Stichtenoth, G.; Jung, P.; Wieg, C.; Kribs, A.; von der Wense, A.; Weller, U.; Höhn, T.; et al. German Neonatal Network. Active perinatal care of preterm infants in the German Neonatal Network. Arch. Dis. Child Fetal Neonatal Ed. 2020, 105, 190-195. [CrossRef] [PubMed]

10. Autran, C.A.; Kellman, B.P.; Kim, J.H.; Asztalos, E.; Blood, A.B.; Spence, E.C.H.; Patel, A.L.; Hou, J.; Lewis, N.E. Human milk oligosaccharide composition predicts risk of necrotising enterocolitis in preterm infants. Gut 2018, 67, 1064-1070. [CrossRef] [PubMed]

11. Narayan, N.R.; Méndez-Lagares, G.; Ardeshir, A.; Lu, D.; Van Rompay, K.K.; Hartigan-O'Connor, D.J. Persistent effects of early infant diet and associated microbiota on the juvenile immune system. Gut Microbes 2015, 6, 284-289. [CrossRef] [PubMed]

12. Zivkovic, A.M.; German, J.B.; Lebrilla, C.B.; Mills, D.A. Human milk glycobiome and its impact on the infant gastrointestinal microbiota. Proc. Natl. Acad. Sci. USA 2011, 108 (Suppl. 1), 4653-4658. [CrossRef] [PubMed]

13. Methods for estimating the due date. Committee Opinion No 700. American College of Obstetricians and Gynecologists. Obstet Gynecol. 2017, 129, 2150-2154.

14. Voigt, M.; Rochow, N.; Straube, S.; Olbertz, D.M.; Jorch, G. Birth weight percentile charts based on daily measurements for very preterm male and female infants at the age of 154-223 days. J. Perinat Med. 2010, 38, 289-295. [CrossRef] [PubMed] 
15. Fenton, T.R.; Kim, J.H. A systematic review and meta-analysis to revise the Fenton growth chart for preterm infants. BMC Pediatrics 2013, 13, 59. [CrossRef] [PubMed]

16. Fenton, T.R. A new growth chart for preterm babies: Babson and Benda's chart updated with recent data and a new format. BMC Pediatr. 2003, 3, 13. [CrossRef]

17. Geffers, C.; Baerwolff, S.; Schwab, F.; Gastmeier, P. Incidence of healthcare- associated infections in high-risk neonates: Results from the German surveillance system for very-low-birthweight infants. J. Hosp. Infect. 2008, 68, 214-221. [CrossRef]

18. Daniels, M.C.; Adair, L.S. Breast-Feeding Influences Cognitive Development in Filipino Children. J. Nutr. 2005, 135, 2589-2595. [CrossRef]

19. Meinzen-Derr, J.; Poindexter, B.; Wrage, L.; Morrow, A.L.; Stoll, B.; Donovan, E.F. Role of human milk in extremely low birth weight infants' risk of necrotizing enterocolitis or death. J. Perinatol. 2009, 29, 57-62. [CrossRef]

20. Sisk, P.M.; Lovelady, C.A.; Dillard, R.G.; Gruber, K.J.; O'Shea, T.M. Early human milk feeding is associated with a lower risk of necrotizing enterocolitis in very low birth weight infants. J. Perinatol. 2007, 27, 428-433. [CrossRef]

21. Corpeleijn, W.E.; Kouwenhoven, S.M.; Paap, M.C.; Van Vliet, I.; Scheerder, I.; Muizer, Y.; Helder, O.K.; van Goudoever, J.B.; Vermeulen, M.J. Intake of own mother's milk during the first days of life is associated with decreased morbidity and mortality in very low birth weight infants during the first 60 days of life. Neonatology 2012, 102, 276-281. [CrossRef] [PubMed]

22. Dewey, K.G. Growth Characteristics of Breast-Fed Compared to Formula-Fed Infants. Biol. Neonate 1998, 74, 94-105. [CrossRef] [PubMed]

23. Spiegler, J.; Preuss, M.; Gebauer, C.; Bendiks, M.; Herting, E.; Goepel, W. Does breastmilk influence the development of bronchopulmonary dysplasia? J. Pediatr. 2016, 169, 76-80 e4. [CrossRef] [PubMed]

24. Battersby, C.; Statnikov, Y.; Santhakumaran, S.; Gray, D.; Modi, N.; Costeloe, K.; UK Neonatal Collaborative and Medicines for Neonates Investigator Group. The United Kingdom National Neonatal Research Database: A validation study. PLoS ONE 2018, 13, e0201815. [CrossRef]

25. Rochow, N.; Landau-Crangle, E.; Fusch, C. Challenges in breast milk fortification for preterm infants. Curr. Opin. Clin. Nutr. Metab. Care 2015, 18, 276-284. [CrossRef]

26. Mills, L.; Modi, N. Clinician enteral feeding preferences for very preterm babies in the UK. Arch. Dis. Child Fetal Neonatal Ed. 2015, 100, F372-F373. [CrossRef]

27. Steenhout, P.; Rochat, F.; Hager, C. The effect of Bifidobacterium lactis on the growth of infants: A pooled analysis of randomized controlled studies. Ann. Nutr. Metab. 2009, 55, 334-340. [CrossRef]

28. Kitajima, H.; Sumida, Y.; Tanaka, R. Early administration of Bifidobacterium breve to preterm infants: Randomized controlled trial. Arch. Dis. Child Fetal Neonatal Ed. 1997, 76, F101-F107. [CrossRef]

29. Sari, F.N.; Eras, Z.; Dizdar, E.A.; Erdeve, O.; Uras, N.; Dilmen, U. Do oral probiotics affect growth and neurodevelopmental outcomes in very low-birth-weight preterm infants? Am. J. Perinatol. 2012, 29, 579-586. [CrossRef]

30. Aceti, A.; Gori, D.; Barone, G.; Callegari, M.L.; Fantini, M.P.; Indrio, F.; Maggio, L.; Meneghin, F.; Morelli, L.; Zuccotti, G.; et al. Probiotics and time to achieve full enteral feeding in human milk-fed and formula-fed preterm infants: Systematic review and meta-analysis. Nutrients 2016, 8, 471. [CrossRef]

31. Sun, J.; Marwah, G.; Westgarth, M.; Buys, N.; Ellwood, D.; Gray, P.H. Effects of Probiotics on Necrotizing Enterocolitis, Sepsis, Intraventricular Hemorrhage, Mortality, Length of Hospital Stay, and Weight Gain in Very Preterm Infants: A Meta-Analysis. Adv. Nutr. 2017, 8, 749-763. [CrossRef] [PubMed]

32. Jacquot, A.; Neveu, D.; Aujoulat, F.; Mercier, G.; Marchandin, H.; Jumas-Bilak, E.; Picaud, J.C. Dynamics and clinical evolution of bacterial gut microflora in extremely premature patients. J. Pediatr. 2011, 158, 390-396. [CrossRef] [PubMed]

33. Aceti, A.; Maggio, L.; Beghetti, I.; Gori, D.; Barone, G.; Callegari, M.; Fantini, M.P.; Indrio, F.; Meneghin, F.; Morelli, L.; et al. Probiotics Prevent Late-Onset Sepsis in Human Milk-Fed, Very Low Birth Weight Preterm Infants: Systematic Review and Meta-Analysis. Nutrients 2017, 9, 904. [CrossRef] [PubMed]

34. Heinemann, A.S.; Pirr, S.; Fehlhaber, B.; Mellinger, L.; Burgmann, J.; Busse, M.; Ginzel, M.; Friesenhagen, J.; von Köckritz-Blickwede, M.; Ulas, T.; et al. In neonates S100A8/S100A9 alarmins prevent the expansion of a specific inflammatory monocyte population promoting septic shock. FASEB J. 2017, 31, 1153-1164. [CrossRef] [PubMed] 
35. Underwood, M.A.; German, J.B.; Lebrilla, C.B.; Mills, D.A. Bifidobacterium longum subspecies infantis: Champion colonizer of the infant gut. Pediatr. Res. 2015, 77, 229-235. [CrossRef] [PubMed]

36. Härtel, C.; Pagel, J.; Spiegler, J.; Buma, J.; Henneke, P.; Zemlin, M.; Viemann, D.; Gille, C.; Gehring, S.; Frommhold, D.; et al. Lactobacillus acidophilus/Bifidobacterium infantis probiotics are associated with increased growth of VLBWI among those exposed to antibiotics. Sci. Rep. 2017, 7, 5633. [CrossRef]

37. Marißen, J.; Haiß, A.; Meyer, C.; Van Rossum, T.; Bünte, L.M.; Frommhold, D.; Gille, C.; Goedicke-Fritz, S.; Göpel, W.; Hudalla, H. Efficacy of Bifidobacterium longum, B. infantis and Lactobacillus acidophilus probiotics to prevent gut dysbiosis in preterm infants of 28+0-32+6 weeks of gestation: A randomised, placebo-controlled, double-blind, multicentre trial: The PRIMAL Clinical Study protocol. BMJ Open 2019, 9, e032617. [CrossRef]

(C) 2020 by the authors. Licensee MDPI, Basel, Switzerland. This article is an open access article distributed under the terms and conditions of the Creative Commons Attribution (CC BY) license (http://creativecommons.org/licenses/by/4.0/). 